\title{
2 Instrumente zur systematischen Erfassung von Verbesserungspotenzialen - Nutzen und Vorgehen einer Selbstbewertung mit Fallbeispiel
}

Christine Kuch

Die Zielsetzung einer auf Lernen und Entwicklung fokussierenden Selbstbewertung besteht zunächst darin, dass man sich innerhalb der Organisation einen systematischen Überblick darüber verschafft, wo man im Augenblick in Bezug auf zuvor festgelegte Merkmale steht. Das mag lapidar klingen - schließlich weiß man doch, wie und wo man arbeitet. In der Arbeitsroutine wird jedoch das eine oder andere übersehen, und so können Selbstbewertungen überraschend blinde Flecken aufdecken.

Die Selbstbewertung kann anhand zuvor selbst festgelegter Qualitätsziele erfolgen (s. Kap. I.1), anhand derer man prüfen kann, inwieweit die eigenen Ziele bereits erreicht sind: Haben alle Mitarbeiter im Verlauf des letzten Jahres Gelegenheit zu einer externen Fortbildung bekommen? Sind während aller Teambesprechungen Protokolle angefertigt worden und wurden diese immer am Anfang der folgenden Sitzung überprüft? Sind sämtliche Beschwerden von Patienten gesammelt worden und wurden sie in gesonderten Treffen bearbeitet?

Man kann für eine Selbstbewertung auch auf vorhandene Selbstbewertungssysteme zurückgreifen (s. Anhang 3, Übersicht über QM-Systeme). Es empfiehlt sich dabei zu prüfen, inwieweit die in den einzelnen Systemen formulierten Merkmale zu den eigenen Vorstellungen guter Qualität passen, da sich diese unterscheiden: So wird beispielsweise in einer Selbstbewertung 
nach dem EFQM-Modell auch nach den Ergebnissen einer Zufriedenheitsbefragung von Mitarbeitern oder Patienten gefragt, während alle anderen Systeme nur den Fokus darauf setzen, ob solche Befragungen überhaupt durchgeführt werden.

In einer Selbstbewertung nach dem QEP-Zielkatalog wird auch die Beschreibung von Behandlungspfaden abgefragt (mindestens 5 Behandlungspfade müssen hier beschrieben werden), während dies sonst nur in einer Selbstbewertung nach dem Katalog der Joint Commission gefragt wird (Informationen hierzu s. Link).

Einer der wichtigsten Vorteile einer solchen lernorientierten Selbstbewertung besteht darin, dass man unter Ausschluss der Öffentlichkeit einen „schonungslos" klaren Blick auf die eigene Organisation werfen kann. Darüber hinaus weiß man als Mitglied der Organisation, wo es in der Organisation „klemmen“ könnte und ist mit diesem Detailwissen jedem OrganisationsFremden, der eine Bewertung durchführt, weit überlegen.

Nehmen an einer Selbstbewertung einer Praxis zudem (fast) alle Mitarbeiter und Praxisinhaber teil, werden, sozusagen nebenbei, unterschiedliche Perspektiven ausgetauscht und eine Diskussion über relevante Qualitätsfragen kann leicht initiiert werden. Dementsprechend sind auch die Akzeptanz und das Vertrauen der Mitarbeiter in die Validität der Ergebnisse bei einer Selbstbewertung höher anzusiedeln als bei einer Fremdbewertung. Die Durchführung einer Selbstbewertung hat ein enormes Potenzial, nachfolgende Qualitätsverbesserungen anzuregen.

\section{Aufgabe 6 für anwendungsfreudige Leser}

Nutzen Sie eine Teamsitzung, an der alle Ärzte und Mitarbeiterinnen teilnehmen, dafür, auf der Basis Ihrer formulierten Qualitätsziele (vgl. Aufgabe 5) zu bewerten: Wo stehen wir in Bezug auf die einzelnen Bereiche? Es empfiehlt sich, dass eine Mitarbeiterin einen Bogen mit den infrage kommenden Zielbereichen vorbereitet. Dieser wird zu Beginn verteilt, und jeder Teilnehmer nimmt eine individuelle Einschätzung vor. Wichtig dabei: Es geht hier um subjektive Sichtweisen, es gibt keine falschen Antworten! Anschließend werden die individuellen Einschätzungen offen ausgetauscht. Ermuntern Sie auch die Schüchternen, sich offen zu beteiligen. Zum Schluss stellen Sie zusammen: Wo stehen wir ganz gut da? Wo können wir uns verbessern? Was ist als nächstes zu tun?

Einer der Nachteile einer (ehrlichen) Selbstbewertung besteht folglich darin, dass sie sich meist kaum für Marketingzwecke eignet. Zudem kann die Validität positiver Ergebnisse in der (Fach-)Öffentlichkeit leicht angezweifelt werden.

Als relevanter Erfolgsfaktor für eine gelungene Selbstbewertung kann auch die von Pfaff (2004) beschriebene Fehlerkultur gelten: So führt die Aufdeckung von Mängeln zwar nicht mehr zu den mittelalterlichen physischen Konse- 
quenzen, die „Boten schlechter Nachrichten“ zu erleiden hatten, wohl aber müssen Mitarbeiter bei einer unzureichenden Fehlerkultur mit entsprechenden verbalen Attacken rechnen. Mitarbeiter, die davon ausgehen müssen, für Fehlerberichte an den Pranger gestellt zu werden (oder andere Kollegen an den Pranger stellen zu müssen), werden sich diesem Risiko kaum aussetzen. Insofern ist der Erfolg einer Selbstbewertung mit dem Ziel, schon Erreichtes herauszufinden und Verbesserungsoptionen $z u$ entdecken, eng an die bestehende Führungskultur geknüpft.

Schließlich fehlt in der Selbstbewertung per definitionem die Außenperspektive, die Lernvorgänge in Bereichen anstoßen kann, an die man bislang kaum gedacht hat. Um diesen Mangel auszugleichen sind reine Selbstbewertungsverfahren oft an Benchmarking- bzw. Referenz-Systeme (s. Kap. III.6) gekoppelt. So kann man beispielsweise bei der EFQM erfahren, wie viele Punkte andere Unternehmen im Schnitt erzielen, und sich damit vergleichen. Bei Nutzung der Internationalen Maturity Matrix (s. Linksammlung), einem neuen, auf europäischer Ebene entwickelten Selbstbewertungsinstrument, wird die Selbstbewertung ebenfalls mit einem Benchmarking-Prozess verknüpft. Die Praxis erhält Rückmeldung dazu, wie sie in Bezug auf die zur Bewertung stehenden Dimensionen im Vergleich zum Durchschnitt und zu den besten der anderen teilnehmenden Praxen steht.

Eine Fremdbewertung, auf die an anderer Stelle (s. Kap. I.6) detaillierter eingegangen wird, dient in aller Regel der Kontrolle. Sei dies die Kontrolle der Einhaltung gesetzlicher Richtlinien oder, wie im Falle einer Zertifizierung, die Kontrolle der für das angestrebte Zertifikat erforderlichen Kriterien. Obwohl eine Fremdbewertung eine Reihe von Vorteilen bringen kann, ist sie in Bezug auf das hier avisierte Ziel des Lernens der Organisation eher nachteilig zu bewerten. Insbesondere bei Zertifizierungen kann beobachtet werden, dass vor der Zertifizierung eifrige „QM-Betriebsamkeit“ besteht, die nach der Zertifizierung zum Erliegen kommt: „Wir haben doch jetzt das Zertifikat!“ Dieser Effekt ist zwar psychologisch verständlich, aber in Hinblick auf ein kontinuierliches Lernen der Organisation eher dysfunktional.

Intelligente Fremdbewertungssysteme sind daher mit anderen, auf Lernen zielende Verfahren, gekoppelt. Ein solches System ist beispielsweise im „Berliner Transfusionsmodell“ verwirklicht. Hier wurde eine gesetzlich vorgeschriebene Kontrollaufsicht als Selbst- und Fremdbewertungsverfahren, an das ein kollegialer Austausch der beteiligten Ärzte geknüpft ist, übersetzt. Das Verfahren ist in Kapitel VI.3 näher beschrieben.

Um eine Selbstbewertung in der Praxis möglichst optimal zu gestalten, sollten die folgenden Schritte beachtet werden:

\subsection{Ablauf einer Selbstbewertung}

1. Die Leitung ist überzeugt vom Nutzen einer Selbstbewertung und möchte damit organisationales Lernen anstoßen. Das bedeutet beispielsweise ganz 
konkret, dass eine Mitarbeiterin für berichtete Missstände nicht gemaßregelt, sondern ihr für den Bericht gedankt wird. Oder auch, dass die leitende Ärztin auf einen Missstand aufmerksam macht, für den sie selbst mitverantwortlich ist.

2. Ein zu den eigenen Qualitätsvorstellungen passendes Selbstbewertungssystem wird ausgewählt und beschafft bzw. es wird selbst entwickelt

3. Ablauf und Verantwortlichkeiten für die anstehende Selbstbewertung werden festgelegt: Sind alle an der Bewertung beteiligt? Wer dokumentiert? Wer fasst die Daten zusammen? Was passiert mit den Ergebnissen? Wie wird nach der Selbstbewertung weiter vorgegangen?

4. Die Mitarbeiter werden über die anstehende Selbstbewertung informiert: Zielsetzung, Inhalte, Ablauf, Verantwortlichkeiten etc.

5. Die Selbstbewertung wird durchgeführt, z.B. an einem Mittwochnachmittag. Gegebenenfalls kann dabei eine externe, neutrale Moderation hilfreich sein, damit der Prozess unter Beteiligung aller Anwesenden, und zugleich effizient, durchgeführt werden kann und beispielsweise auch gegensätzliche Meinungen frei geäußert werden können.

6. Die Ergebnisse der Selbstbewertung werden zusammengefasst und im gesamten Team besprochen: Wo klappt es gut? An welchen Stellen besteht Verbesserungsbedarf? Was sollten wir zuerst ändern?

7. Ein Handlungsplan wird im Team entwickelt und umgesetzt.

8. Nach einer zuvor festgesetzten Zeit werden die umgesetzten Veränderungen evaluiert.

9. Gegebenenfalls werden nun noch Anpassungen vorgenommen, ansonsten wird der nächste Veränderungsbereich identifiziert, ein neuer Handlungsplan wird umgesetzt, evaluiert usw.

10. Die Selbstbewertung wird jährlich wiederholt, um erneut einen Blick über die gesamte Organisation zu erlangen und gegebenenfalls neue Handlungsfelder zu entdecken.

Die Durchführung einer Selbstbewertung wird nachstehend an einem Praxisbeispiel veranschaulicht.

\section{Praxisbeispiel: Selbstbewertung und Benchmarking mit der Internationalen Maturity Matrix}

Hausarztpraxen, die am Pilotprojekt der Internationalen Maturity Matrix (IMM, s. Linksammlung) teilnahmen, nahmen Kontakt mit der Leitung der von der Kassenärztlichen Bundesvereinigung mitgeförderten deutschen Pilotstudie auf. Die Projektleitung klärte die Praxisinhaber darüber auf, dass die IMM in einem interaktiven Prozess zwischen Wissenschaftlern und Praktikern aus 12 europäischen Ländern entwickelt wurde und nun zur Erprobung, unter anderem in Deutschland, eingesetzt wird. Das Instrument zielt darauf, Weiterentwicklungsprozesse in Praxen anzustoßen. Es besteht aus sieben Dimensionen, denen jeweils eine sechsstufige Skala unterlegt ist, die einen zunehmenden organisationalen Entwicklungsstand einer Arztpraxis indizieren. Die Dimensio- 
2 Instrumente zur systematischen Erfassung von Verbesserungspotenzialen Nutzen und Vorgehen einer Selbstbewertung mit Fallbeispiel

nen der IMM, denen eine systematische Übersichtsarbeit zu organisationalen Erfolgsfaktoren und deren Abstimmung mit Praktikern zugrunde liegt (Tapp et al. 2008), lauten wie folgt:

- Informationsbeschaffung und -nutzung

- Patientenunterlagen und Dokumentation

- Mitarbeiterführung und -orientierung

- Arbeit als Team

- Patientenorientierung

- Qualitätsverbesserung

- interne Regelungen und Ablaufbeschreibungen

Die Selbstbewertung mit der IMM beinhaltet eine eineinhalbstündige, extern moderierte Gruppensitzung des gesamten Praxisteams, d. h. neben Arzt bzw. Ärztin nehmen auch Med. Fachangestellte und weiteres Praxispersonal an der Sitzung teil. Im Rahmen der Sitzung füllt zunächst jeder Teilnehmende die IMM alleine aus, d. h. jeder schätzt ein, auf welcher der 6 Stufen die Praxis in Bezug auf die einzelne Dimensionen liegt. Im zweiten Schritt wird im Rahmen einer moderierten Diskussion eine gemeinsame Einschätzung im Konsens entwickelt. In einer nachfolgenden zweiten, etwa einstündigen Sitzung erhält die Praxis von der Moderatorin sogenannte Benchmarking-Ergebnisse: Das Konsensergebnis der Praxis wird in Bezug gesetzt zum Durchschnittsergebnis aller und zum Ergebnis der besten teilnehmenden Praxen. Auf diesem Vergleich aufbauend wählt das Praxisteam einen verbesserungswürdigen Bereich aus und erstellt gemeinsam mit der Moderatorin einen Aktionsplan zur Weiterentwicklung der Praxis.

Einige Rückmeldungen teilnehmender Praxen verdeutlichen, dass auch eine solche kurze und pragmatische Intervention deutlichen Nutzen für die Praxen ergeben kann:

„Ich bin sicher, für unsere Praxis ist daraus ein guter weiterer Gang in Richtung fortlaufender Selbstbewertung und gemeinsamer Problemlösung möglich. " und „Sowohl vom eingehaltenen Zeitrahmen als auch vom inhaltlichen Anspruch und Ergebnis für uns und auch von der atmosphärischen Stimmung, so haben es mich die Anderen wissen lassen, gibt es nur positive Rückmeldungen. Mir persönlich hat besonders gut gefallen, wie alle Beteiligten von ihren jeweils teilweise sehr unterschiedlichen Voraussetzungen abgeholt wurden und alle in den Diskussionsprozess integriert wurden. "Dr. med. Johannes Hauswaldt, Hannover

„Es war sehr angenehm und es gab eine fruchtbare Diskussion in der Praxis. Die Beteiligten waren sehr zufrieden. "Dr. med. Johann Warns, Varel

„In der Praxis hat sich als ein Ergebnis der IMM folgendes ergeben. Die Helferinnen treffen sich nun regelmäßig (ca. 1-mal/Monat) unabhängig von den Teambesprechungen. Auf den Treffen der Helferinnen werden zum einen zwischenmenschliche Spannungen etc. besprochen, die Helferinnen erarbeiten jedoch auch unter sich Arbeitsabläufe und Vorgehensweisen, um ihre Arbeit 
besser auf einander abstimmen zu können. Dies hat bereits zu deutlich weniger Reibungsverlusten (vor allem, da ja alle Helferinnen mehr oder weniger als Teilzeitkräfte arbeiten) geführt. Ein für mich positives Ergebnis. Hier hat sich die Teilnahme an der Pilotstudie auf jeden Fall gelohnt." Dr. Uwe Kurzke, Pellworm

\section{Literatur}

Pfaff H, Ernstmann N, Pritzbuer E von: Das Fehlerkultur-Modell: Warum gibt es im Krankenhaus keine Fehlerkultur? Gesundheitsökonomie \& Qualitätsmanagement 2004; 9/5: 271-273

Tapp L, Edwards A, Braspenning I, Grol R, Eriksson T, Kuch C, Elwyn G: Developing the International Family Practice Maturity Matrix - an organisational assessment tool for primary care. Project report 2008

\section{Links}

Informationen zur Internationalen Maturity Matrix

www.maturitymatrix.co.uk, Interessenten an der IMM können sich auch gerne an die Autorin, Frau Dr. Christine Kuch, wenden (kuch@medcoaching.de).

Informationen zum Qualitätszielkatalog von QEP

http://www.kbv.de/qep/qep.html

Informationen zum Katalog der Joint commission http://www.jointcommission.org/AccreditationPrograms/ AmbulatoryCare/ 